\section{Introduction to Automated Particle Analysis}

T.B. Vander Wood, MVA, Inc.

A microscopist is presented with a special problem when asked to characterize a population of particles. In order to adequately describe the sample, it may be necessary to examine hundreds or even thousands of individual particles, recording the size, shape, identity or other parameters of interest. To manually characterize a statistically significant fraction of the sample could require many hours of tedious work at the scope, followed by still more tedious hours of data reduction. What is needed is a technique to automate the repetitive parts of this process: the recognition and characterization of particles in the sample and the reduction of data and presentation of results. This technique is automated particle analysis.

In 1968, automated particle analysis (APA) took early, tentative steps when Moore and coworkers at what was then the National Bureau of Standards demonstrated the possibility of computer analysis of images of small particles.' The computing power needed for this work was massive for the day and restricted growth of the field for ten years, but in 1980 , publications by Lee and others ${ }^{2,3}$ and Fritz et al. ${ }^{4}$ described the first commercially available automated particle analysis system for scanning electron microscopes. This system found and analyzed particles on the fly, without acquiring images of the particles, and although supplanted by new techniques, is still in use today. With the widespread availability of powerful and inexpensive desktop computers and digital image acquisition, APA has come of age, giving any microscopist the ability to size and characterize thousands of individual particles in a reasonable amount of time.

Automated particle analysis is in many ways a specialized application of the broader collection of techniques known as image analysis, ${ }^{5}$ and is most often found as part of an image analysis package. These packages are available from a variety of vendors for light and particle beam based microscopies or they can be assembled from off-the-shelf components. Over the past few years applications in fields ranging from air pollution situdies to superconductor fabrication have been published. ${ }^{6-11}$

The front end of an APA system consists of a digitizer that can present an image of particles to the computer. In light microscopy and TEMs the image is usually recorded by the high tech equivalent of a home video camera, with a frame grabber mounted in the computer to record the image. In scanning microscopies (SEMs, STEMs, STMs, FIBs, etc.) a digitized image is built up by recording signal intensity as a correlate to the $x, y$ position of the beam on the sample. In either case the result is a two dimensional array with a signal intensity stored in each cell, which when displayed on film or a CRT, is seen as an image. But it is a two dimensional array to the computer and it is the ability of computers to manipulate large data arrays that makes APA possible.

Once the image is in computer memory, the possibilities for manipulation are nearly endless. ${ }^{12}$ Brightness and contrast may be adjusted, features sharpened or blurred, and touching features separated. Automation of these image processing functions is possible in most software packages, but automation must be applied with caution since variations in the input image can cause unpredictable results. Processing of the image prior to particle measurement can be a powerful aid to analysis but is best applied with human supervision at least until the stability of the processing results is established.

Particles are recognized (by the computer) in the image (matrix) as a set of contiguous pixels (cells) with brightness (values) above a threshold set by the analyst. Alternatively, particles may be recognized as dark features against a bright background, or as features with brightness between two predefined intensity levels. Once the particles have been recognized and defined, any two dimensional morphological parameter can be measured and recorded for each particle. The most common are diameters and aspect ratio, but others may be important. If the analysis is carried out in an analytical instrument (e.g. an SEM with EDS), then analytical information for each particle can also be recorded. These measurements and analyses can be carried out for thousands of particles in a matter of hours, and may result in data sets with tens of thousands of data points.

With the large data sets involved, automation of data reduction is essential. Usually the data will be imported into a statistical or spreadsheet software package. These packages allow determination of summary statistics for the measured parameters and displays of parameter distributions. Particles may also be classified into types based on the recorded data, either by standard database functions or statistical techniques ${ }_{1}^{13}$ and the measured parameters for each type reported separately.

Automated particle analysis brings to microscopy the advantages of light scattering particle measurement systems (the ability to make large numbers of measurements in a short time) while allowing for the significant advantages of microscopy: the ability to make real (rather than inferred) measurement, in two dimensions (rather than one), while discriminating among particles of different types.

G.A. Moore et al, in R.T. DeHoff and F.N. Rhines, eds., Quantitative Microscopy, New York: McGraw Hill (1968) 381

2. R.J. Lee and R.M. Fisher, NBS Spec. Publ. 533 (1980) 63

3. J.F. Kelly et al., SEM/1980, I(1980) 311

4. G.S. Fritz et al., in R.H. Geiss, ed., Microbeam Analysis-1981, San Francisco: San Francisco Press, (1981) 57.

5. J.C. Russ, Computer Assisted Microscopy, New York Plenum Press, (1990).

6. M.S. Germani and P.R. Buseck, Anal. Chem, 63 (1991) 2232.

7. M.S. Germani, Jour For. Sci, 36 (1991) 331.

8. T.B. Vander Wood and L.D. Detter-Hoskin, in K.L. Mittal, ed., Proceedings of the Second Symposium on Particles in Gases and Liquids, New York: Plenum Press, (1990).

9. T.B. Vander Wood and W.R. Boltin, in D.M. Hausen, et al., eds., Process Mineralogy XI, TMS (1991) 37

10. T.B. Vander Wood, Proc. Ann. EMSA Meeting 50 (1992) 1492

11. J. Hefter and L. Steriti, Part. Sci. and Tech., 10 (1992) 91

12. J.C. Russ, The image Processing Handbook, Boca Raton: CRC Press, (1992)

13. T.W. Shattuck et al., Anal. Chem., 63 (1991) 2646.

Reprinted from Proceedings, 52nd Annual Meeting of the Microscopy Society of America.

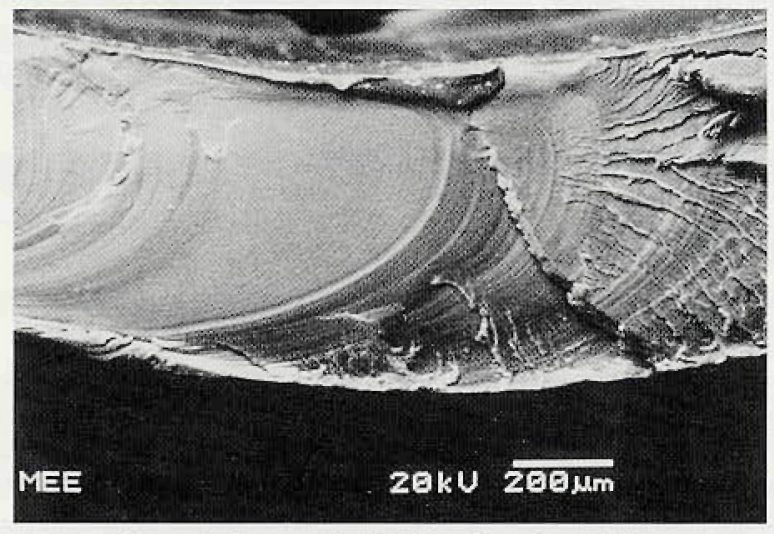

Figure 3: Progressive fracture of a polymer tube.

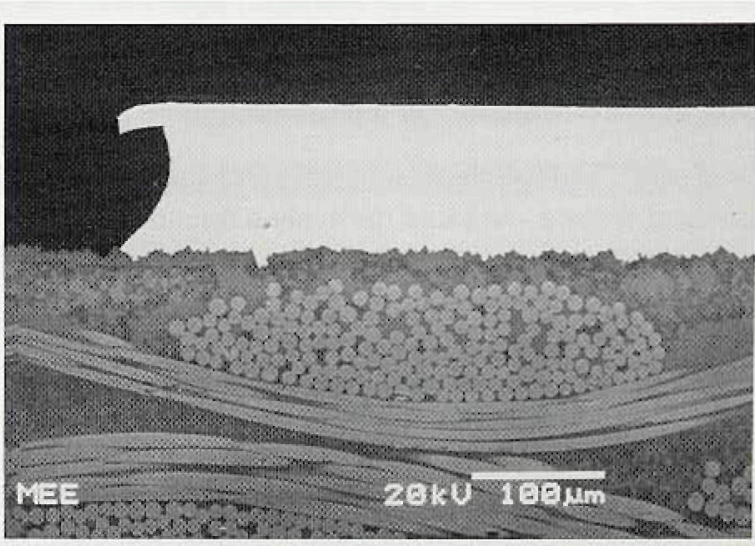

Figure 4: Polished cross section of PC board. 